\title{
APPENDIX 12
}

\section{Volatile Organic Compounds (VOC) Emission Rates}

VOC emissions by trees are estimated based on procedures used in the EPA's Biogenic Emissions Inventory System (BEIS) (U.S. EPA 2017a). The VOC emissions depend on tree species, leaf biomass, air temperature, and other environmental factors. Table 30 shows the base genusspecific emission factors used in i-Tree. These values are averages from the literature. If genusspecific information is not available, median emission values for the family, order, or superorder are used to represent the genus or family. These emission values are standardized to $30{ }^{\circ} \mathrm{C}$ and photosynthetically active radiation (PAR) flux of $1,000 \mu \mathrm{mol} / \mathrm{m}^{2} / \mathrm{s}$.

Table 30.-Base VOC emission rates ( $\mu \mathrm{g} / \mathrm{g}$ leaf dry weight/hour) for isoprenes and monoterpenes, by genus

\begin{tabular}{|l|c|c|}
\hline Genus & Isoprene & Monoterpene \\
\hline Abarema & 9.313 & 0.327 \\
\hline Abelia & 0.000 & 0.000 \\
\hline Abies & 2.300 & 3.000 \\
\hline Abutilon & 0.000 & 0.000 \\
\hline Acacia & 0.031 & 2.440 \\
\hline Acalypha & 0.067 & 0.133 \\
\hline Acca & 28.083 & 0.500 \\
\hline Acer & 0.017 & 1.338 \\
\hline Achyranthes & 6.648 & 0.114 \\
\hline Acnistus & 0.078 & 0.120 \\
\hline Acoelorrhaphe & 18.180 & 0.080 \\
\hline Acokanthera & 0.100 & 0.200 \\
\hline Acosmium & 9.313 & 0.327 \\
\hline Acradenia & 0.100 & 1.450 \\
\hline Acrocomia & 18.180 & 0.080 \\
\hline Actinidia & 0.086 & 0.157 \\
\hline Actinostemon & 0.067 & 0.133 \\
\hline Adansonia & 0.000 & 0.000 \\
\hline Adelia & 0.067 & 0.133 \\
\hline Adenanthera & 9.313 & 0.327 \\
\hline Adenostoma & 0.000 & 0.360 \\
\hline Adolphia & 12.833 & 1.067 \\
\hline Adonidia & 0.100 & 0.080 \\
\hline Aegiphila & 0.100 & 0.100 \\
\hline Aeonium & 0.048 & 0.028 \\
\hline
\end{tabular}

\begin{tabular}{|l|r|c|}
\hline Genus & Isoprene & Monoterpene \\
\hline Aesculus & 0.333 & 0.200 \\
\hline Afrocarpus & 0.000 & 0.000 \\
\hline Afrormosia & 9.313 & 0.327 \\
\hline Agarista & 0.086 & 0.157 \\
\hline Agathis & 0.100 & 1.500 \\
\hline Ageratina & 0.100 & 2.428 \\
\hline Agonis & 28.083 & 0.500 \\
\hline Agrostis & 4.400 & 0.510 \\
\hline Aidia & 0.000 & 0.000 \\
\hline Ailanthus & 0.000 & 0.000 \\
\hline Aiouea & 0.083 & 0.167 \\
\hline Aiphanes & 18.180 & 0.080 \\
\hline Alberta & 0.000 & 0.000 \\
\hline Albizia & 40.000 & 0.200 \\
\hline Alchornea & 0.067 & 0.133 \\
\hline Alchorneopsis & 0.067 & 0.133 \\
\hline Alectryon & 44.900 & 0.000 \\
\hline Aleurites & 0.000 & 0.000 \\
\hline Alhagi & 9.313 & 0.327 \\
\hline Allamanda & 0.100 & 0.200 \\
\hline Allocasuarina & 70.000 & 0.100 \\
\hline Allophylus & 44.900 & 0.000 \\
\hline Allosyncarpia & 28.083 & 0.500 \\
\hline Alloxylon & 0.100 & 0.200 \\
\hline Alnus & 0.005 & 5.390 \\
\hline & & $c 0 n t i n u e d$ \\
\hline
\end{tabular}

Appendix 12 (https://doi.org/10.2737/NRS-GTR-200-2021-Appendix12) in: Nowak, David J. 2021. Understanding i-Tree: Summary of programs and methods. Gen. Tech. Rep. NRS-200-2021. Madison, WI: U.S. Department of Agriculture, Forest Service, Northern Research Station. 100 p. [plus 14 appendixes]. https://doi.org/10.2737/NRS-GTR-200-2021. 
Table 30.-Continued

\begin{tabular}{|c|c|c|}
\hline Genus & Isoprene & Monoterpene \\
\hline Aloe & 15.150 & 0.068 \\
\hline Aloysia & 0.100 & 0.100 \\
\hline Alphitonia & 12.833 & 1.067 \\
\hline Alseis & 0.000 & 0.000 \\
\hline Alsophila & 6.424 & 0.068 \\
\hline Alstonia & 0.100 & 0.200 \\
\hline Alvaradoa & 0.000 & 0.000 \\
\hline Amaioua & 0.000 & 0.000 \\
\hline Amelanchier & 0.000 & 0.000 \\
\hline Amelasorbus & 0.057 & 0.029 \\
\hline Amherstia & 9.313 & 0.327 \\
\hline Amorpha & 9.313 & 0.327 \\
\hline Ampelopsis & 0.000 & 0.000 \\
\hline Amphipappus & 0.100 & 2.428 \\
\hline Amphitecna & 0.275 & 0.075 \\
\hline Amyris & 0.003 & 0.000 \\
\hline Anacardium & 0.000 & 2.260 \\
\hline Anadenanthera & 9.313 & 0.327 \\
\hline Andira & 9.313 & 0.327 \\
\hline Angophora & 28.083 & 0.500 \\
\hline Aniba & 0.083 & 0.167 \\
\hline Anisacanthus & 0.118 & 0.091 \\
\hline Annona & 0.000 & 0.000 \\
\hline Anopterus & 6.741 & 0.415 \\
\hline Anthocephalus & 0.000 & 0.000 \\
\hline Antidaphne & 6.741 & 0.415 \\
\hline Antidesma & 0.067 & 0.133 \\
\hline Antiphytum & 0.000 & 0.000 \\
\hline Antirhea & 0.000 & 0.000 \\
\hline Aparisthmium & 0.067 & 0.133 \\
\hline Apeiba & 0.000 & 0.000 \\
\hline Apophyllum & 6.648 & 0.114 \\
\hline Apuleia & 9.313 & 0.327 \\
\hline Aralia & 0.100 & 0.200 \\
\hline Araucaria & 0.000 & 1.500 \\
\hline Arbutus & 0.050 & 0.093 \\
\hline Archirhodomyrts & 28.083 & 0.500 \\
\hline Archontophoenix & 18.180 & 0.080 \\
\hline Arctostaphylos & 0.000 & 0.000 \\
\hline Ardisia & 6.648 & 0.114 \\
\hline
\end{tabular}

\begin{tabular}{|c|c|c|}
\hline Genus & Isoprene & Monoterpene \\
\hline Arecastrum & 18.180 & 0.080 \\
\hline Argusia & 0.000 & 0.000 \\
\hline Argyrautia & 0.100 & 2.428 \\
\hline Argyrodendron & 0.000 & 0.000 \\
\hline Argyroxiphium & 0.100 & 2.428 \\
\hline Aristotelia & 0.000 & 0.000 \\
\hline Artemisia & 0.500 & 23.600 \\
\hline Arthrocarpum & 9.927 & 0.327 \\
\hline Artocarpus & 8.625 & 0.133 \\
\hline Asimina & 0.000 & 0.000 \\
\hline Aspidosperma & 0.100 & 0.200 \\
\hline Asteromyrtus & 28.083 & 0.500 \\
\hline Astrocaryum & 18.180 & 0.080 \\
\hline Astronium & 0.000 & 2.260 \\
\hline Atalaya & 44.900 & 0.000 \\
\hline Atamisquea & 6.648 & 0.114 \\
\hline Atherosperma & 0.083 & 0.167 \\
\hline Athrotaxis & 0.086 & 0.714 \\
\hline Atriplex & 0.500 & 3.000 \\
\hline Attalea & 18.180 & 0.080 \\
\hline Aucuba & 0.100 & 1.600 \\
\hline Auerodendron & 12.833 & 1.067 \\
\hline Aureliana & 0.078 & 0.120 \\
\hline Austrocedrus & 0.086 & 0.714 \\
\hline Averrhoa & 6.741 & 0.415 \\
\hline Avicennia & 0.000 & 0.000 \\
\hline Ayenia & 0.000 & 0.000 \\
\hline Azadirachta & 0.100 & 0.100 \\
\hline Azara & 0.100 & 0.100 \\
\hline Azolla & 3.730 & 0.068 \\
\hline Baccharis & 0.000 & 2.428 \\
\hline Backhousia & 28.083 & 0.500 \\
\hline Bactris & 18.180 & 0.080 \\
\hline Balizia & 9.313 & 0.327 \\
\hline Bambusa & 0.002 & 0.006 \\
\hline Banara & 0.100 & 0.100 \\
\hline Banisteriopsis & 0.010 & 0.010 \\
\hline Banksia & 0.100 & 0.200 \\
\hline Barleria & 0.118 & 0.091 \\
\hline Barleriola & 0.118 & 0.091 \\
\hline
\end{tabular}


Table 30.-Continued

\begin{tabular}{|c|c|c|}
\hline Genus & Isoprene & Monoterpene \\
\hline Barringtonia & 6.648 & 0.114 \\
\hline Batesimalva & 0.000 & 0.000 \\
\hline Bathysa & 0.000 & 0.000 \\
\hline Bauhinia & 9.313 & 0.327 \\
\hline Beaucarnea & 15.150 & 0.068 \\
\hline Bedfordia & 0.100 & 2.428 \\
\hline Befaria & 0.086 & 0.157 \\
\hline Beilschmiedia & 0.083 & 0.167 \\
\hline Berberis & 22.200 & 0.000 \\
\hline Bernardia & 0.067 & 0.133 \\
\hline Betula & 0.000 & 1.395 \\
\hline Bidens & 0.100 & 2.428 \\
\hline Bischofia & 0.100 & 0.200 \\
\hline Bismarckia & 18.180 & 0.080 \\
\hline Bixa & 0.100 & 0.100 \\
\hline Blepharocalyx & 28.083 & 0.500 \\
\hline Blighia & 44.900 & 0.000 \\
\hline Bobea & 0.000 & 0.000 \\
\hline Bocconia & 0.083 & 0.167 \\
\hline Boehmeria & 4.971 & 0.150 \\
\hline Bolusanthus & 9.313 & 0.327 \\
\hline Bombax & 0.000 & 0.000 \\
\hline Bontia & 0.118 & 0.091 \\
\hline Borrichia & 0.100 & 2.428 \\
\hline Bouchea & 0.100 & 0.100 \\
\hline Bougainvillea & 0.100 & 0.100 \\
\hline Bourreria & 0.000 & 0.000 \\
\hline Bouvardia & 0.000 & 0.000 \\
\hline Brachychiton & 0.000 & 0.000 \\
\hline Brahea & 18.180 & 0.080 \\
\hline Brassaia & 0.100 & 0.200 \\
\hline Breynia & 0.067 & 0.133 \\
\hline Brighamia & 0.078 & 0.620 \\
\hline Brongniartia & 9.313 & 0.327 \\
\hline Brosimum & 8.625 & 0.133 \\
\hline Broussaisia & 0.048 & 0.200 \\
\hline Broussonetia & 11.500 & 0.133 \\
\hline Brugmansia & 0.000 & 0.120 \\
\hline Bruguiera & 0.100 & 0.100 \\
\hline Brunellia & 0.048 & 0.028 \\
\hline
\end{tabular}

\begin{tabular}{|c|c|c|}
\hline Genus & Isoprene & Monoterpene \\
\hline Brunfelsia & 0.078 & 0.120 \\
\hline Brya & 9.313 & 0.327 \\
\hline Buchanania & 0.000 & 2.260 \\
\hline Buchenavia & 5.550 & 0.050 \\
\hline Bucida & 5.550 & 0.050 \\
\hline Buckinghamia & 0.100 & 0.200 \\
\hline Buckleya & 6.741 & 0.415 \\
\hline Buddleja & 0.118 & 0.091 \\
\hline Bumelia & 0.000 & 0.000 \\
\hline Bunchosia & 0.010 & 0.010 \\
\hline Bursaria & 0.000 & 0.000 \\
\hline Butia & 18.180 & 0.080 \\
\hline Buxus & 13.500 & 0.067 \\
\hline Byrsonima & 0.010 & 0.010 \\
\hline Cabralea & 0.100 & 0.100 \\
\hline Caesalpinia & 0.000 & 0.327 \\
\hline Cajanus & 9.313 & 0.327 \\
\hline Callaeum & 0.010 & 0.010 \\
\hline Calliandra & 9.313 & 0.327 \\
\hline Callicarpa & 0.100 & 0.100 \\
\hline Callicoma & 0.048 & 0.028 \\
\hline Callistemon & 7.500 & 0.000 \\
\hline Callitris & 0.086 & 0.714 \\
\hline Calluna & 0.000 & 1.610 \\
\hline Calocedrus & 0.000 & 0.600 \\
\hline Calodendrum & 0.100 & 1.450 \\
\hline Calophyllum & 11.900 & 0.000 \\
\hline Calotropis & 0.100 & 0.200 \\
\hline Calpurnia & 9.313 & 0.327 \\
\hline Calycanthus & 0.000 & 0.167 \\
\hline Calycophyllum & 0.000 & 0.000 \\
\hline Calyptranthes & 28.083 & 0.500 \\
\hline Calyptronoma & 18.180 & 0.080 \\
\hline Calytrix & 28.083 & 0.500 \\
\hline Camellia & 0.000 & 0.000 \\
\hline Campomanesia & 28.083 & 0.500 \\
\hline Camptotheca & 70.000 & 0.600 \\
\hline Cananga & 0.000 & 0.000 \\
\hline Canarium & 8.456 & 1.100 \\
\hline Canella & 2.660 & 1.200 \\
\hline
\end{tabular}


Table 30.-Continued

\begin{tabular}{|c|c|c|}
\hline Genus & Isoprene & Monoterpene \\
\hline Canotia & 0.100 & 0.200 \\
\hline Canthium & 0.000 & 0.000 \\
\hline Capparis & 6.648 & 0.114 \\
\hline Caragana & 0.100 & 0.200 \\
\hline Carallia & 0.100 & 0.100 \\
\hline Carica & 0.000 & 0.100 \\
\hline Cariniana & 6.648 & 0.114 \\
\hline Carissa & 0.000 & 0.000 \\
\hline Carmona & 0.000 & 0.000 \\
\hline Carnegia & 0.100 & 0.100 \\
\hline Carpenteria & 0.000 & 0.028 \\
\hline Carpinus & 0.000 & 0.040 \\
\hline Carpobrotus & 0.100 & 0.100 \\
\hline Carpotroche & 6.741 & 0.415 \\
\hline Carya & 0.000 & 0.700 \\
\hline Caryodendron & 0.067 & 0.133 \\
\hline Caryopteris & 0.100 & 0.100 \\
\hline Caryota & 18.180 & 0.080 \\
\hline Casasia & 0.000 & 0.000 \\
\hline Casearia & 0.100 & 0.100 \\
\hline Casimiroa & 0.100 & 1.450 \\
\hline Cassia & 0.000 & 0.327 \\
\hline Cassine & 0.100 & 0.200 \\
\hline Cassipourea & 0.100 & 0.100 \\
\hline Castanea & 0.333 & 6.930 \\
\hline Castanopsis & 0.000 & 0.000 \\
\hline Castanospermum & 9.313 & 0.327 \\
\hline Castela & 0.000 & 0.000 \\
\hline Castilla & 0.000 & 0.133 \\
\hline Casuarina & 70.000 & 0.100 \\
\hline Catalpa & 0.000 & 0.200 \\
\hline Catesbaea & 0.000 & 0.000 \\
\hline Ceanothus & 0.000 & 3.595 \\
\hline Cecropia & 0.000 & 0.150 \\
\hline Cedrela & 0.100 & 0.100 \\
\hline Cedrus & 0.000 & 1.000 \\
\hline Ceiba & 0.000 & 0.000 \\
\hline Celastrus & 0.100 & 0.200 \\
\hline Celosia & 6.648 & 0.114 \\
\hline Celtis & 0.000 & 0.200 \\
\hline
\end{tabular}

\begin{tabular}{|c|c|c|}
\hline Genus & Isoprene & Monoterpene \\
\hline Centrolobium & 9.313 & 0.327 \\
\hline Cephalanthus & 0.000 & 0.000 \\
\hline Cephalotaxus & 0.086 & 1.700 \\
\hline Ceratonia & 0.000 & 1.000 \\
\hline Ceratopetalum & 0.048 & 0.028 \\
\hline Cercidiphyllum & 56.900 & 1.600 \\
\hline Cercidium & 0.100 & 0.200 \\
\hline Cercis & 0.010 & 0.000 \\
\hline Cercocarpus & 0.333 & 0.100 \\
\hline Cereus & 0.100 & 0.100 \\
\hline Cestrum & 0.000 & 0.120 \\
\hline Chaenomeles & 0.000 & 0.000 \\
\hline Chamaebatia & 0.057 & 0.029 \\
\hline Chamaebatiaria & 0.057 & 0.029 \\
\hline Chamaecrista & 9.313 & 0.327 \\
\hline Chamaecyparis & 0.000 & 0.200 \\
\hline Chamaecytisus & 9.313 & 0.327 \\
\hline Chamaedaphne & 0.000 & 0.157 \\
\hline Chamaedorea & 18.180 & 0.080 \\
\hline Chamaerops & 18.180 & 0.080 \\
\hline Chamaesyce & 0.067 & 0.133 \\
\hline Charpentiera & 6.648 & 0.114 \\
\hline Cheirodendron & 0.100 & 0.200 \\
\hline Chenopodium & 0.100 & 0.100 \\
\hline Chilopsis & 0.000 & 0.100 \\
\hline Chiococca & 0.000 & 0.000 \\
\hline Chionanthus & 0.033 & 0.117 \\
\hline Chione & 0.000 & 0.000 \\
\hline Chitalpa & 0.275 & 0.075 \\
\hline Choisya & 0.100 & 1.450 \\
\hline Choricarpia & 28.083 & 0.500 \\
\hline Chorisia & 0.000 & 0.000 \\
\hline Chromolaena & 0.100 & 2.428 \\
\hline Chrysalidocarpus & 18.180 & 0.080 \\
\hline Chrysanthemum & 0.000 & 0.300 \\
\hline Chrysobalanus & 0.048 & 0.028 \\
\hline Chrysolepis & 0.000 & 0.267 \\
\hline Chrysophyllum & 0.000 & 0.000 \\
\hline Chrysothamnus & 0.100 & 2.428 \\
\hline Cibotium & 6.424 & 0.068 \\
\hline
\end{tabular}


Table 30.-Continued

\begin{tabular}{|c|c|c|}
\hline Genus & Isoprene & Monoterpene \\
\hline Cinchona & 0.000 & 0.000 \\
\hline Cinnamomum & 0.000 & 0.030 \\
\hline Citharexylum & 0.100 & 0.100 \\
\hline Citronella & 0.078 & 0.620 \\
\hline Citrullus & 0.100 & 0.100 \\
\hline Citrus & 0.000 & 0.737 \\
\hline Cladrastis & 0.100 & 0.200 \\
\hline Claoxylon & 0.067 & 0.133 \\
\hline Clarisia & 8.625 & 0.133 \\
\hline Clermontia & 0.078 & 0.620 \\
\hline Clerodendrum & 0.100 & 0.100 \\
\hline Clethra & 6.648 & 0.114 \\
\hline Cleyera & 0.000 & 0.000 \\
\hline Clibadium & 0.100 & 2.428 \\
\hline Clidemia & 5.550 & 0.050 \\
\hline Cliftonia & 14.000 & 0.100 \\
\hline Clinopodium & 0.100 & 5.000 \\
\hline Clusia & 0.000 & 0.000 \\
\hline Cnemidaria & 6.424 & 0.068 \\
\hline Cneoridium & 0.100 & 1.450 \\
\hline Cnidoscolus & 0.067 & 0.133 \\
\hline Coccoloba & 0.000 & 0.100 \\
\hline Coccothrinax & 18.180 & 0.080 \\
\hline Cocculus & 28.133 & 0.067 \\
\hline Cochlospermum & 0.100 & 0.100 \\
\hline Cocos & 18.180 & 0.080 \\
\hline Codariocalyx & 9.313 & 0.327 \\
\hline Codiaeum & 0.067 & 0.133 \\
\hline Codonocarpus & 6.741 & 0.415 \\
\hline Coffea & 0.000 & 0.000 \\
\hline Cojoba & 9.313 & 0.327 \\
\hline Coleogyne & 0.057 & 0.029 \\
\hline Colubrina & 0.050 & 1.067 \\
\hline Colutea & 9.313 & 0.327 \\
\hline Colvillea & 9.313 & 0.327 \\
\hline Comarostaphylis & 0.000 & 0.157 \\
\hline Commersonia & 0.000 & 0.000 \\
\hline Comocladia & 0.000 & 2.260 \\
\hline Condalia & 12.833 & 1.067 \\
\hline Conocarpus & 11.000 & 0.000 \\
\hline
\end{tabular}

\begin{tabular}{|c|c|c|}
\hline Genus & Isoprene & Monoterpene \\
\hline Conostegia & 5.550 & 0.050 \\
\hline Conradina & 0.100 & 5.000 \\
\hline Copaifera & 9.313 & 0.327 \\
\hline Copernicia & 18.180 & 0.080 \\
\hline Coprosma & 0.000 & 0.000 \\
\hline Corchorus & 0.000 & 0.000 \\
\hline Cordia & 0.000 & 0.000 \\
\hline Cordyline & 15.150 & 0.068 \\
\hline Cornus & 0.000 & 1.600 \\
\hline Cornutia & 0.100 & 0.100 \\
\hline Corokia & 0.100 & 0.100 \\
\hline Coronilla & 9.313 & 0.327 \\
\hline Corylopsis & 44.350 & 3.000 \\
\hline Corylus & 0.000 & 0.000 \\
\hline Corymbia & 28.083 & 0.500 \\
\hline Corynocarpus & 0.100 & 0.200 \\
\hline Corypha & 18.180 & 0.080 \\
\hline Cotinus & 0.000 & 0.000 \\
\hline Cotoneaster & 0.000 & 0.000 \\
\hline Couepia & 0.048 & 0.028 \\
\hline Couratari & 6.648 & 0.114 \\
\hline Couroupita & 6.648 & 0.114 \\
\hline Coursetia & 9.313 & 0.327 \\
\hline Coussarea & 0.000 & 0.000 \\
\hline Coutarea & 0.000 & 0.000 \\
\hline Cowania & 0.057 & 0.029 \\
\hline Crataegus & 0.000 & 4.610 \\
\hline Crescentia & 0.275 & 0.075 \\
\hline Crinodendron & 0.000 & 0.000 \\
\hline Critonia & 0.100 & 2.428 \\
\hline Critoniopsis & 0.100 & 2.428 \\
\hline Crossopetalum & 0.000 & 0.200 \\
\hline Crossosoma & 0.048 & 0.028 \\
\hline Crotalaria & 9.313 & 0.327 \\
\hline Croton & 11.065 & 1.484 \\
\hline Cryptocarya & 0.083 & 0.167 \\
\hline Cryptomeria & 0.000 & 3.000 \\
\hline Cudrania & 8.625 & 0.133 \\
\hline Cunninghamia & 0.000 & 3.000 \\
\hline Cupania & 44.900 & 0.000 \\
\hline
\end{tabular}


Table 30.-Continued

\begin{tabular}{|c|c|c|}
\hline Genus & Isoprene & Monoterpene \\
\hline Cupaniopsis & 44.900 & 0.000 \\
\hline Cupressocyparis & 0.100 & 0.600 \\
\hline Cupressus & 0.000 & 0.900 \\
\hline Cussonia & 0.100 & 0.200 \\
\hline Cuttsia & 0.100 & 0.100 \\
\hline Cyanea & 0.078 & 0.620 \\
\hline Cyathea & 6.424 & 0.068 \\
\hline Cybianthus & 6.648 & 0.114 \\
\hline Cybistax & 0.275 & 0.075 \\
\hline Cycas & 0.000 & 0.068 \\
\hline Cyclobalanopsis & 0.100 & 0.400 \\
\hline Cydonia & 0.057 & 0.029 \\
\hline Cynometra & 9.313 & 0.327 \\
\hline Cypholophus & 4.971 & 0.150 \\
\hline Cyphomandra & 0.078 & 0.120 \\
\hline Cyrilla & 14.000 & 0.100 \\
\hline Cyrtandra & 0.118 & 0.620 \\
\hline Cytisus & 37.000 & 0.002 \\
\hline Dacryodes & 8.456 & 1.100 \\
\hline Dahlia & 0.100 & 2.428 \\
\hline Dahlstedtia & 9.927 & 0.327 \\
\hline Dais & 5.550 & 0.050 \\
\hline Dalbergia & 9.313 & 0.327 \\
\hline Dalea & 9.313 & 0.327 \\
\hline Daphne & 5.550 & 0.050 \\
\hline Daphnopsis & 5.550 & 0.050 \\
\hline Dasiphora & 0.057 & 0.029 \\
\hline Davidia & 42.000 & 0.600 \\
\hline Davidsonia & 0.048 & 0.028 \\
\hline Dedeckera & 0.100 & 0.100 \\
\hline Deeringothamnus & 0.000 & 0.000 \\
\hline Delissea & 0.078 & 0.620 \\
\hline Delonix & 0.000 & 0.200 \\
\hline Dendrocalamus & 0.002 & 0.006 \\
\hline Dendromecon & 0.083 & 0.167 \\
\hline Dendropanax & 0.100 & 0.200 \\
\hline Dendropemon & 6.741 & 0.415 \\
\hline Dendrophthora & 6.741 & 0.415 \\
\hline Denhamia & 0.100 & 0.200 \\
\hline Derris & 9.313 & 0.327 \\
\hline
\end{tabular}

\begin{tabular}{|c|c|c|}
\hline Genus & Isoprene & Monoterpene \\
\hline Deutzia & 0.048 & 0.028 \\
\hline Diatenopteryx & 44.900 & 0.000 \\
\hline Dichrostachys & 0.000 & 0.327 \\
\hline Dicksonia & 6.424 & 0.068 \\
\hline Dictyoloma & 0.100 & 1.450 \\
\hline Diervilla & 0.000 & 0.000 \\
\hline Dillenia & 0.000 & 0.114 \\
\hline Diospyros & 0.000 & 0.100 \\
\hline Dipholis & 0.000 & 0.000 \\
\hline Diphysa & 9.313 & 0.327 \\
\hline Diplacus & 0.000 & 0.000 \\
\hline Dipterocarpus & 0.000 & 0.000 \\
\hline Dipteronia & 44.900 & 0.000 \\
\hline Dirca & 5.550 & 0.050 \\
\hline Ditta & 0.067 & 0.133 \\
\hline Dodonaea & 44.900 & 0.000 \\
\hline Dombeya & 0.000 & 0.000 \\
\hline Doryphora & 0.083 & 0.167 \\
\hline Dovyalis & 0.100 & 0.100 \\
\hline Dracaena & 15.150 & 0.068 \\
\hline Drimys & 0.000 & 1.200 \\
\hline Drypetes & 0.067 & 0.133 \\
\hline Dubautia & 0.100 & 2.428 \\
\hline Duranta & 0.100 & 0.100 \\
\hline Dypsis & 18.180 & 0.080 \\
\hline Dysoxylum & 0.100 & 0.100 \\
\hline Ebenopsis & 9.313 & 0.327 \\
\hline Ecclinusa & 0.000 & 0.000 \\
\hline Echium & 0.000 & 0.000 \\
\hline Edgeworthia & 5.550 & 0.050 \\
\hline Ehretia & 0.000 & 0.000 \\
\hline Elaeagnus & 0.000 & 0.200 \\
\hline Elaeocarpus & 0.000 & 0.000 \\
\hline Elaeodendron & 0.100 & 0.200 \\
\hline Eleutherococcus & 0.100 & 0.200 \\
\hline Elliottia & 0.086 & 0.157 \\
\hline Embelia & 6.648 & 0.114 \\
\hline Embothrium & 0.100 & 0.200 \\
\hline Emorya & 0.118 & 0.091 \\
\hline Encelia & 0.100 & 6.000 \\
\hline
\end{tabular}


Table 30.-Continued

\begin{tabular}{|c|c|c|}
\hline Genus & Isoprene & Monoterpene \\
\hline Endiandra & 0.083 & 0.167 \\
\hline Endlicheria & 0.083 & 0.167 \\
\hline Entada & 9.313 & 0.327 \\
\hline Entelea & 0.000 & 0.000 \\
\hline Enterolobium & 0.000 & 0.327 \\
\hline Epilobium & 5.550 & 0.050 \\
\hline Eranthemum & 0.118 & 0.091 \\
\hline Eremophila & 0.000 & 0.000 \\
\hline Erica & 0.086 & 0.157 \\
\hline Ericameria & 0.500 & 3.000 \\
\hline Eriobotrya & 0.000 & 2.520 \\
\hline Eriodictyon & 0.078 & 0.120 \\
\hline Eriotheca & 0.000 & 0.000 \\
\hline Erithalis & 0.000 & 0.000 \\
\hline Ernodea & 0.000 & 0.000 \\
\hline Erythrina & 9.313 & 0.327 \\
\hline Erythrophleum & 9.313 & 0.327 \\
\hline Erythroxylum & 0.000 & 0.415 \\
\hline Escallonia & 0.000 & 0.000 \\
\hline Esenbeckia & 0.100 & 1.450 \\
\hline Eubrachion & 6.741 & 0.415 \\
\hline Eucalyptus & 37.446 & 3.798 \\
\hline Eucommia & 19.700 & 0.800 \\
\hline Eucryphia & 0.048 & 0.028 \\
\hline Eugenia & 10.200 & 0.000 \\
\hline Euonymus & 0.100 & 0.200 \\
\hline Euphorbia & 0.000 & 0.133 \\
\hline Euplassa & 0.100 & 0.200 \\
\hline Eupomatia & 2.660 & 1.200 \\
\hline Euroschinus & 0.000 & 2.260 \\
\hline Eurya & 0.000 & 0.000 \\
\hline Euryops & 0.000 & 2.428 \\
\hline Euterpe & 18.180 & 0.080 \\
\hline Exocarpos & 6.741 & 0.415 \\
\hline Exochorda & 0.057 & 0.029 \\
\hline Exostema & 0.000 & 0.000 \\
\hline Exothea & 44.900 & 0.000 \\
\hline Eysenhardtia & 9.313 & 0.327 \\
\hline Fagraea & 0.100 & 0.200 \\
\hline Fagus & 0.000 & 0.210 \\
\hline
\end{tabular}

\begin{tabular}{|c|c|c|}
\hline Genus & Isoprene & Monoterpene \\
\hline Falcataria & 9.313 & 0.327 \\
\hline Fallugia & 0.057 & 0.029 \\
\hline Faramea & 0.000 & 0.000 \\
\hline Fatsia & 0.100 & 0.200 \\
\hline Feijoa & 28.083 & 0.500 \\
\hline Fendlera & 0.048 & 0.028 \\
\hline Fendlerella & 0.048 & 0.028 \\
\hline Ferocactus & 0.100 & 0.100 \\
\hline Ficus & 6.973 & 0.850 \\
\hline Filicium & 44.900 & 0.000 \\
\hline Firmiana & 0.000 & 0.000 \\
\hline Fitchia & 0.100 & 2.428 \\
\hline Fitzroya & 0.086 & 0.714 \\
\hline Flacourtia & 0.100 & 0.100 \\
\hline Flindersia & 0.100 & 1.450 \\
\hline Flourensia & 0.100 & 2.428 \\
\hline Floydia & 0.100 & 0.200 \\
\hline Flueggea & 0.067 & 0.133 \\
\hline Fontanesia & 0.033 & 0.117 \\
\hline Forchhammeria & 6.648 & 0.114 \\
\hline Forestiera & 0.000 & 0.117 \\
\hline Forsythia & 0.000 & 0.100 \\
\hline Fortunella & 0.100 & 1.450 \\
\hline Fothergilla & 44.350 & 3.000 \\
\hline Fouquieria & 0.000 & 0.120 \\
\hline Frangula & 10.170 & 0.630 \\
\hline Franklinia & 0.000 & 0.000 \\
\hline Fraxinus & 0.000 & 0.000 \\
\hline Fremontodendron & 0.000 & 0.000 \\
\hline Fuchsia & 5.550 & 0.050 \\
\hline Funtumia & 0.100 & 0.200 \\
\hline Furcraea & 15.150 & 0.068 \\
\hline Galium & 0.000 & 0.000 \\
\hline Gallesia & 0.100 & 0.100 \\
\hline Ganophyllum & 44.900 & 0.000 \\
\hline Garberia & 0.100 & 2.428 \\
\hline Garcinia & 0.000 & 0.000 \\
\hline Gardenia & 0.000 & 0.000 \\
\hline Garrya & 0.000 & 1.100 \\
\hline Gastrolobium & 9.313 & 0.327 \\
\hline
\end{tabular}


Table 30.-Continued

\begin{tabular}{|c|c|c|}
\hline Genus & Isoprene & Monoterpene \\
\hline Gaussia & 18.180 & 0.080 \\
\hline Gaylussacia & 0.086 & 0.157 \\
\hline Geijera & 0.100 & 1.450 \\
\hline Gelsemium & 0.100 & 0.200 \\
\hline Genipa & 0.000 & 0.000 \\
\hline Genista & 9.313 & 0.327 \\
\hline Genistidium & 9.313 & 0.327 \\
\hline Geonoma & 18.180 & 0.080 \\
\hline Geranium & 0.100 & 0.200 \\
\hline Gesneria & 0.118 & 0.091 \\
\hline Ginkgo & 1.000 & 3.000 \\
\hline Ginoria & 0.000 & 0.000 \\
\hline Gleditsia & 0.000 & 0.000 \\
\hline Gliricidia & 9.313 & 0.327 \\
\hline Glochidion & 6.741 & 0.415 \\
\hline Glossopetalon & 0.048 & 0.028 \\
\hline Glycosmis & 0.100 & 1.450 \\
\hline Gmelina & 0.100 & 0.100 \\
\hline Gochnatia & 0.100 & 2.428 \\
\hline Goetzea & 0.078 & 0.120 \\
\hline Gomidesia & 28.083 & 0.500 \\
\hline Gompholobium & 9.313 & 0.327 \\
\hline Gonocalyx & 0.086 & 0.157 \\
\hline Gonzalagunia & 0.000 & 0.000 \\
\hline Goodia & 9.313 & 0.327 \\
\hline Gordonia & 0.000 & 0.000 \\
\hline Gossypium & 0.020 & 0.600 \\
\hline Gouania & 12.833 & 1.067 \\
\hline Graffenrieda & 5.550 & 0.050 \\
\hline Graptophyllum & 0.118 & 0.091 \\
\hline Grevillea & 0.000 & 0.200 \\
\hline Grewia & 0.000 & 0.000 \\
\hline Griselinia & 21.050 & 1.100 \\
\hline Guaiacum & 8.456 & 1.100 \\
\hline Guapira & 0.100 & 0.100 \\
\hline Guarea & 0.100 & 0.100 \\
\hline Guatteria & 0.000 & 0.000 \\
\hline Guazuma & 0.000 & 0.000 \\
\hline Guettarda & 0.000 & 0.000 \\
\hline Guioa & 44.900 & 0.000 \\
\hline
\end{tabular}

\begin{tabular}{|c|c|c|}
\hline Genus & Isoprene & Monoterpene \\
\hline Gundlachia & 0.100 & 2.428 \\
\hline Gyminda & 0.100 & 0.200 \\
\hline Gymnanthes & 0.011 & 0.000 \\
\hline Gymnocladus & 0.000 & 0.000 \\
\hline Gymnostoma & 70.000 & 0.100 \\
\hline Haematoxylum & 9.313 & 0.327 \\
\hline Haenianthus & 0.033 & 0.117 \\
\hline Hakea & 0.100 & 0.200 \\
\hline Halesia & 0.000 & 0.000 \\
\hline Halimodendron & 9.313 & 0.327 \\
\hline Hamamelis & 1.000 & 0.200 \\
\hline Hamelia & 0.000 & 0.000 \\
\hline Handroanthus & 0.275 & 0.075 \\
\hline Harpephyllum & 0.000 & 2.260 \\
\hline Harpullia & 44.900 & 0.000 \\
\hline Harrisia & 0.100 & 0.100 \\
\hline Havardia & 9.313 & 0.327 \\
\hline Hazardia & 0.100 & 2.428 \\
\hline Hebe & 0.000 & 0.000 \\
\hline Hedycarya & 0.083 & 0.167 \\
\hline Hedyosmum & 0.083 & 0.167 \\
\hline Hedyotis & 0.000 & 0.000 \\
\hline Heimia & 0.000 & 0.000 \\
\hline Heisteria & 6.741 & 0.415 \\
\hline Helichrysum & 0.100 & 2.428 \\
\hline Helicteres & 0.000 & 0.000 \\
\hline Helietta & 0.100 & 1.450 \\
\hline Heliocarpus & 0.000 & 0.000 \\
\hline Hemizonia & 0.100 & 2.428 \\
\hline Henriettea & 5.550 & 0.050 \\
\hline Heritiera & 0.000 & 0.000 \\
\hline Hernandia & 0.083 & 0.167 \\
\hline Hesperomannia & 0.100 & 2.428 \\
\hline Heterocondylus & 0.100 & 2.428 \\
\hline Heteromeles & 0.000 & 0.000 \\
\hline Heteropterys & 0.010 & 0.010 \\
\hline Heterotrichum & 5.550 & 0.050 \\
\hline Hibiscadelphus & 0.000 & 0.000 \\
\hline Hibiscus & 0.000 & 0.000 \\
\hline Hillia & 0.000 & 0.000 \\
\hline
\end{tabular}


Table 30.-Continued

\begin{tabular}{|c|c|c|}
\hline Genus & Isoprene & Monoterpene \\
\hline Himatanthus & 0.100 & 0.200 \\
\hline Hippomane & 0.067 & 0.133 \\
\hline Hippophae & 0.100 & 0.200 \\
\hline Hirtella & 0.048 & 0.028 \\
\hline Hoheria & 0.000 & 0.000 \\
\hline Holmskioldia & 0.100 & 0.100 \\
\hline Holocarpha & 0.100 & 3.100 \\
\hline Holodiscus & 0.057 & 0.029 \\
\hline Homalanthus & 0.067 & 0.133 \\
\hline Homalium & 0.100 & 0.100 \\
\hline Hopea & 0.000 & 0.000 \\
\hline Horsfieldia & 13.000 & 1.200 \\
\hline Hovenia & 18.500 & 1.100 \\
\hline Howea & 18.180 & 0.080 \\
\hline Hura & 0.067 & 0.133 \\
\hline Hydrangea & 0.000 & 0.000 \\
\hline Hydriastele & 18.180 & 0.080 \\
\hline Hyeronima & 0.067 & 0.133 \\
\hline Hylocereus & 0.100 & 0.100 \\
\hline Hymenaea & 9.313 & 0.327 \\
\hline Hymenoclea & 0.100 & 2.428 \\
\hline Hymenosporum & 0.000 & 0.000 \\
\hline Hyophorbe & 18.180 & 0.080 \\
\hline Hypelate & 0.000 & 0.000 \\
\hline Hyperbaena & 28.133 & 0.067 \\
\hline Hypericum & 0.000 & 0.000 \\
\hline Hyptis & 0.100 & 5.000 \\
\hline Ilex & 0.000 & 0.000 \\
\hline Illicium & 0.083 & 0.167 \\
\hline Indigofera & 9.313 & 0.327 \\
\hline Inga & 9.313 & 0.327 \\
\hline Iris & 15.150 & 0.068 \\
\hline Isocoma & 0.100 & 2.428 \\
\hline Isodendrion & 0.100 & 0.100 \\
\hline Itea & 0.000 & 0.000 \\
\hline Iva & 0.100 & 0.100 \\
\hline Ixora & 0.000 & 0.000 \\
\hline Jacaranda & 0.000 & 0.000 \\
\hline Jacaratia & 0.100 & 0.100 \\
\hline Jacksonia & 9.313 & 0.327 \\
\hline
\end{tabular}

\begin{tabular}{|l|c|c|}
\hline Genus & Isoprene & Monoterpene \\
\hline Jacquinia & 0.000 & 0.114 \\
\hline Jagera & 44.900 & 0.000 \\
\hline Jamesia & 0.048 & 0.028 \\
\hline Jasminum & 0.033 & 0.117 \\
\hline Jatropha & 0.067 & 0.133 \\
\hline Joannesia & 0.067 & 0.133 \\
\hline Jubaea & 18.180 & 0.080 \\
\hline Juglans & 0.000 & 4.033 \\
\hline Juniperus & 0.000 & 0.700 \\
\hline Justicia & 0.118 & 0.091 \\
\hline Kalmia & 1.000 & 0.200 \\
\hline Kalmiopsis & 0.086 & 0.157 \\
\hline Kalopanax & 0.100 & 0.200 \\
\hline Kanaloa & 9.313 & 0.327 \\
\hline Karwinskia & 12.833 & 1.000 \\
\hline Keckiella & 0.000 & 0.000 \\
\hline Kerria & 0.000 & 0.000 \\
\hline Khaya & 0.000 & 0.000 \\
\hline Kielmeyera & 0.1000 & 0.100 \\
\hline Kigelia & 0.1000 & 0.100 \\
\hline Kleinhovia & 6.741 & 0.415 \\
\hline Koeberlinia & 0.000 & 0.075 \\
\hline Koelreuteria & 0.000 & 0.000 \\
\hline Kokia & 6.648 & 0.114 \\
\hline Kolkwitzia & 0.000 & 0.000 \\
\hline Krameria & 0.000 & 0.000 \\
\hline Krugiodendron & 0.000 & 0.000 \\
\hline Kunzea & 0.010 & 0.010 \\
\hline Labordia & 0.000 \\
\hline Laburnum & 0.000 \\
\hline Lacistema & 0.100 \\
\hline Laetia & 0.000 \\
\hline Lafoensia & 0.000 \\
\hline Lagarostrobos & 0.000 \\
\hline Lagascea & 0.000 \\
\hline Lagerstroemia & 0.000 \\
\hline Laguncularia & 0.000 \\
\hline Lamanonia & 0.000 & 0.000 \\
\hline
\end{tabular}


Table 30.-Continued

\begin{tabular}{|c|c|c|}
\hline Genus & Isoprene & Monoterpene \\
\hline Laplacea & 0.000 & 0.000 \\
\hline Larix & 0.050 & 8.200 \\
\hline Larrea & 0.000 & 1.100 \\
\hline Lasianthus & 0.000 & 0.000 \\
\hline Laurus & 0.000 & 0.930 \\
\hline Lavandula & 0.100 & 5.000 \\
\hline Lawsonia & 0.000 & 0.000 \\
\hline Leandra & 5.550 & 0.050 \\
\hline Lecythis & 6.648 & 0.114 \\
\hline Ledum & 0.000 & 0.157 \\
\hline Leitneria & 13.432 & 0.856 \\
\hline Leonotis & 0.100 & 5.000 \\
\hline Lepidospartum & 0.100 & 2.428 \\
\hline Leptocereus & 0.000 & 0.100 \\
\hline Leptomeria & 6.741 & 0.415 \\
\hline Leptopus & 0.067 & 0.133 \\
\hline Leptospermum & 28.083 & 0.500 \\
\hline Lespedeza & 9.313 & 0.327 \\
\hline Leucadendron & 0.100 & 0.200 \\
\hline Leucaena & 0.050 & 0.427 \\
\hline Leucophyllum & 0.000 & 0.000 \\
\hline Leucothoe & 0.086 & 0.157 \\
\hline Leycesteria & 0.000 & 0.000 \\
\hline Libocedrus & 0.086 & 0.714 \\
\hline Licania & 0.048 & 0.028 \\
\hline Licaria & 0.083 & 0.167 \\
\hline Ligustrum & 70.500 & 0.000 \\
\hline Limonium & 0.100 & 0.100 \\
\hline Lindera & 0.100 & 0.100 \\
\hline Lippia & 0.100 & 0.100 \\
\hline Liquidambar & 50.860 & 2.770 \\
\hline Liriodendron & 2.050 & 0.000 \\
\hline Litchi & 0.000 & 0.000 \\
\hline Lithocarpus & 0.000 & 0.400 \\
\hline Lithraea & 0.000 & 2.260 \\
\hline Lithrea & 0.000 & 2.260 \\
\hline Litsea & 0.083 & 0.167 \\
\hline Livistona & 18.180 & 0.080 \\
\hline Lobelia & 0.078 & 0.620 \\
\hline Lomatia & 0.100 & 0.200 \\
\hline
\end{tabular}

\begin{tabular}{|c|c|c|}
\hline Genus & Isoprene & Monoterpene \\
\hline Lonchocarpus & 9.313 & 0.327 \\
\hline Lonicera & 0.000 & 0.000 \\
\hline Lophomyrtus & 28.083 & 0.500 \\
\hline Lophostemon & 28.083 & 0.500 \\
\hline Luehea & 0.000 & 0.000 \\
\hline Luma & 28.083 & 0.500 \\
\hline Lunania & 0.100 & 0.100 \\
\hline Lycianthes & 0.078 & 0.120 \\
\hline Lycium & 0.078 & 0.120 \\
\hline Lycopersicon & 0.000 & 0.120 \\
\hline Lyonia & 0.086 & 0.157 \\
\hline Lyonothamnus & 0.000 & 0.029 \\
\hline Lysiloma & 0.000 & 0.327 \\
\hline Lysimachia & 6.648 & 0.114 \\
\hline Maackia & 9.313 & 0.327 \\
\hline Macadamia & 0.100 & 0.200 \\
\hline Macaranga & 0.067 & 0.133 \\
\hline Machaerium & 9.313 & 0.327 \\
\hline Machaonia & 0.000 & 0.000 \\
\hline Maclura & 0.000 & 0.000 \\
\hline Macrozamia & 6.424 & 0.068 \\
\hline Maesopsis & 12.833 & 1.067 \\
\hline Magnolia & 0.100 & 6.000 \\
\hline Mahonia & 40.000 & 0.200 \\
\hline Malacomeles & 0.057 & 0.029 \\
\hline Malephora & 0.100 & 0.100 \\
\hline Mallotus & 0.067 & 0.133 \\
\hline Malosma & 0.000 & 2.260 \\
\hline Malouetia & 0.100 & 0.200 \\
\hline Malpighia & 0.010 & 0.010 \\
\hline Malus & 1.000 & 7.425 \\
\hline Malvaviscus & 0.000 & 0.000 \\
\hline Mammea & 0.000 & 0.000 \\
\hline Mangifera & 0.000 & 2.260 \\
\hline Manglietia & 0.100 & 1.600 \\
\hline Manihot & 0.067 & 0.133 \\
\hline Manilkara & 0.000 & 0.000 \\
\hline Mappia & 0.100 & 0.200 \\
\hline Maprounea & 0.067 & 0.133 \\
\hline Maranthes & 0.048 & 0.028 \\
\hline
\end{tabular}


Table 30.-Continued

\begin{tabular}{|c|c|c|}
\hline Genus & Isoprene & Monoterpene \\
\hline Marcgravia & 6.648 & 0.114 \\
\hline Margaritaria & 0.067 & 0.133 \\
\hline Markhamia & 0.275 & 0.075 \\
\hline Marlierea & 28.083 & 0.500 \\
\hline Mastichodendron & 0.000 & 0.000 \\
\hline Matayba & 44.900 & 0.000 \\
\hline Matelea & 0.100 & 0.200 \\
\hline Maytenus & 0.100 & 0.200 \\
\hline Mecranium & 5.550 & 0.050 \\
\hline Medinilla & 5.550 & 0.050 \\
\hline Melaleuca & 22.100 & 0.000 \\
\hline Melastoma & 5.550 & 0.050 \\
\hline Melia & 0.000 & 0.100 \\
\hline Melicoccus & 44.900 & 0.000 \\
\hline Melicope & 0.100 & 1.450 \\
\hline Meliosma & 28.133 & 0.067 \\
\hline Melochia & 0.000 & 0.000 \\
\hline Menodora & 0.033 & 0.117 \\
\hline Menziesia & 0.086 & 0.157 \\
\hline Meriania & 5.550 & 0.050 \\
\hline Meryta & 0.100 & 0.200 \\
\hline Mespilus & 0.057 & 0.029 \\
\hline Metasequoia & 0.000 & 3.000 \\
\hline Metopium & 0.000 & 2.260 \\
\hline Metrodorea & 0.100 & 1.450 \\
\hline Metrosideros & 28.083 & 0.500 \\
\hline Metternichia & 0.078 & 0.120 \\
\hline Michelia & 0.100 & 1.600 \\
\hline Miconia & 0.000 & 0.050 \\
\hline Micropholis & 0.000 & 0.000 \\
\hline Millettia & 9.313 & 0.327 \\
\hline Mimosa & 9.313 & 0.327 \\
\hline Mimusops & 0.000 & 0.000 \\
\hline Mitracarpus & 0.000 & 0.000 \\
\hline Mollinedia & 0.083 & 0.167 \\
\hline Monotoca & 0.086 & 0.157 \\
\hline Montanoa & 0.100 & 2.428 \\
\hline Montrichardia & 18.180 & 0.080 \\
\hline Morella & 0.000 & 1.100 \\
\hline Morinda & 0.000 & 0.000 \\
\hline
\end{tabular}

\begin{tabular}{|c|c|c|}
\hline Genus & Isoprene & Monoterpene \\
\hline Moringa & 6.648 & 0.114 \\
\hline Morisonia & 6.648 & 0.114 \\
\hline Mortonia & 0.100 & 0.200 \\
\hline Morus & 0.000 & 1.600 \\
\hline Mouriri & 5.550 & 0.050 \\
\hline Muehlenbeckia & 0.100 & 0.100 \\
\hline Munroidendron & 0.100 & 0.200 \\
\hline Muntingia & 0.000 & 0.000 \\
\hline Murraya & 0.100 & 1.450 \\
\hline Musa & 0.000 & 0.068 \\
\hline Myoporum & 0.118 & 0.091 \\
\hline Myrceugenia & 28.083 & 0.500 \\
\hline Myrcia & 28.083 & 0.500 \\
\hline Myrcianthes & 28.083 & 0.500 \\
\hline Myrciaria & 28.083 & 0.500 \\
\hline Myrica & 0.100 & 2.400 \\
\hline Myristica & 0.000 & 1.200 \\
\hline Myrocarpus & 9.313 & 0.327 \\
\hline Myrospermum & 9.313 & 0.327 \\
\hline Myroxylon & 9.313 & 0.327 \\
\hline Myrsine & 6.648 & 0.114 \\
\hline Myrtus & 80.950 & 0.150 \\
\hline Nandina & 22.550 & 0.000 \\
\hline Nauclea & 0.000 & 0.000 \\
\hline Nectandra & 0.083 & 0.167 \\
\hline Neea & 0.100 & 0.100 \\
\hline Nematolepis & 0.100 & 1.450 \\
\hline Nemopanthus & 0.100 & 0.200 \\
\hline Neolamarckia & 0.000 & 0.000 \\
\hline Neolaugeria & 0.000 & 0.000 \\
\hline Neomitranthes & 28.083 & 0.500 \\
\hline Neraudia & 4.971 & 0.150 \\
\hline Nerium & 0.000 & 0.000 \\
\hline Nesoluma & 0.000 & 0.000 \\
\hline Nestegis & 0.033 & 0.117 \\
\hline Nestronia & 6.741 & 0.415 \\
\hline Neviusia & 0.057 & 0.029 \\
\hline Nicotiana & 0.078 & 0.120 \\
\hline Nierembergia & 0.078 & 0.120 \\
\hline Nolina & 0.000 & 0.068 \\
\hline
\end{tabular}


Table 30.-Continued

\begin{tabular}{|c|c|c|}
\hline Genus & Isoprene & Monoterpene \\
\hline Notelaea & 0.033 & 0.117 \\
\hline Nothocestrum & 0.078 & 0.120 \\
\hline Nothofagus & 0.000 & 0.267 \\
\hline Nototrichium & 6.648 & 0.114 \\
\hline Nuytsia & 6.741 & 0.415 \\
\hline Nypa & 18.180 & 0.080 \\
\hline Nyssa & 77.000 & 0.600 \\
\hline Ochna & 0.000 & 0.000 \\
\hline Ochroma & 0.000 & 0.000 \\
\hline Ochrosia & 0.100 & 0.200 \\
\hline Ocotea & 0.083 & 0.167 \\
\hline Odontonema & 0.118 & 0.091 \\
\hline Oemleria & 0.057 & 0.029 \\
\hline Olea & 0.000 & 0.190 \\
\hline Olearia & 0.100 & 2.428 \\
\hline Olneya & 9.313 & 0.327 \\
\hline Ononis & 9.313 & 0.327 \\
\hline Oplonia & 0.118 & 0.091 \\
\hline Oplopanax & 0.100 & 0.200 \\
\hline Opuntia & 0.000 & 0.100 \\
\hline Orbignya & 18.180 & 0.080 \\
\hline Ormosia & 9.313 & 0.327 \\
\hline Ornithostaphyls & 0.086 & 0.157 \\
\hline Osmanthus & 0.000 & 0.100 \\
\hline Osteomeles & 0.057 & 0.029 \\
\hline Ostrya & 0.000 & 0.000 \\
\hline Ottoschulzia & 0.100 & 0.200 \\
\hline Ouratea & 0.000 & 0.000 \\
\hline Oxandra & 0.000 & 0.000 \\
\hline Oxydendrum & 0.000 & 0.600 \\
\hline Oxylobium & 9.313 & 0.327 \\
\hline Pachira & 0.000 & 0.000 \\
\hline Pachycereus & 0.100 & 0.100 \\
\hline Pachystroma & 0.067 & 0.133 \\
\hline Paederia & 0.000 & 0.000 \\
\hline Paeonia & 13.432 & 0.856 \\
\hline Palaquium & 0.000 & 0.000 \\
\hline Palicourea & 0.000 & 0.000 \\
\hline Paliurus & 12.833 & 1.067 \\
\hline Pandanus & 18.180 & 0.080 \\
\hline
\end{tabular}

\begin{tabular}{|c|c|c|}
\hline Genus & Isoprene & Monoterpene \\
\hline Parapiptadenia & 9.313 & 0.327 \\
\hline Paraserianthes & 9.313 & 0.327 \\
\hline Parathesis & 6.648 & 0.114 \\
\hline Parkia & 9.313 & 0.327 \\
\hline Parkinsonia & 0.000 & 0.327 \\
\hline Parmentiera & 0.275 & 0.075 \\
\hline Parrotia & 44.350 & 3.000 \\
\hline Parryella & 9.313 & 0.327 \\
\hline Parthenium & 0.100 & 2.428 \\
\hline Parthenocissus & 0.000 & 0.000 \\
\hline Patagonula & 0.000 & 0.000 \\
\hline Paulownia & 0.000 & 0.000 \\
\hline Paxistima & 0.100 & 0.200 \\
\hline Pedilanthus & 0.067 & 0.133 \\
\hline Peltogyne & 9.313 & 0.327 \\
\hline Peltophorum & 9.313 & 0.327 \\
\hline Pentzia & 0.100 & 2.428 \\
\hline Pera & 0.067 & 0.133 \\
\hline Peraphyllum & 0.057 & 0.029 \\
\hline Pereskia & 0.100 & 0.100 \\
\hline Perrottetia & 0.100 & 0.200 \\
\hline Persea & 0.000 & 1.100 \\
\hline Persoonia & 0.100 & 0.200 \\
\hline Petalostigma & 6.741 & 0.415 \\
\hline Petalostylis & 9.313 & 0.327 \\
\hline Petitia & 0.100 & 0.100 \\
\hline Peucephyllum & 0.100 & 2.428 \\
\hline Peumus & 0.083 & 0.167 \\
\hline Phaulothamnus & 0.100 & 0.100 \\
\hline Phellodendron & 0.000 & 1.600 \\
\hline Phialanthus & 0.000 & 0.000 \\
\hline Philadelphus & 0.000 & 0.000 \\
\hline Phillyrea & 0.033 & 0.117 \\
\hline Phlomis & 0.100 & 5.000 \\
\hline Phoenix & 7.500 & 0.000 \\
\hline Photinia & 0.000 & 0.000 \\
\hline Phyllanthus & 0.000 & 0.133 \\
\hline Phyllocladus & 0.086 & 1.700 \\
\hline Phyllostachys & 0.002 & 0.006 \\
\hline Phyllostemonodaphne & 0.083 & 0.167 \\
\hline
\end{tabular}


Table 30.-Continued

\begin{tabular}{|c|c|c|}
\hline Genus & Isoprene & Monoterpene \\
\hline Physalis & 0.078 & 0.120 \\
\hline Physocarpus & 0.057 & 0.029 \\
\hline Phytolacca & 0.000 & 0.100 \\
\hline Picea & 6.748 & 2.692 \\
\hline Pickeringia & 9.313 & 0.327 \\
\hline Picramnia & 0.000 & 0.000 \\
\hline Picrasma & 0.000 & 0.000 \\
\hline Pictetia & 36.829 & 0.000 \\
\hline Pieris & 0.100 & 0.100 \\
\hline Piloblephis & 0.100 & 5.000 \\
\hline Pilocarpus & 0.100 & 1.450 \\
\hline Pilosocereus & 0.000 & 0.100 \\
\hline Pimenta & 0.000 & 0.000 \\
\hline Pinckneya & 0.000 & 0.000 \\
\hline Pinus & 0.000 & 3.276 \\
\hline Piper & 0.083 & 0.167 \\
\hline Piptadenia & 9.313 & 0.327 \\
\hline Piptocarpha & 0.100 & 2.428 \\
\hline Piptocoma & 0.100 & 2.428 \\
\hline Pipturus & 4.971 & 0.150 \\
\hline Piscidia & 9.313 & 0.327 \\
\hline Pisonia & 0.100 & 0.100 \\
\hline Pistacia & 0.000 & 8.370 \\
\hline Pithecellobium & 9.313 & 0.327 \\
\hline Pittosporum & 0.000 & 0.000 \\
\hline Planchonella & 0.000 & 0.000 \\
\hline Planera & 0.100 & 0.167 \\
\hline Platanus & 29.658 & 0.027 \\
\hline Plathymenia & 9.313 & 0.327 \\
\hline Platycladus & 0.086 & 0.714 \\
\hline Platycyamus & 9.313 & 0.327 \\
\hline Platydesma & 0.100 & 1.450 \\
\hline Platymiscium & 9.313 & 0.327 \\
\hline Plectranthus & 0.100 & 5.000 \\
\hline Pleodendron & 2.660 & 1.200 \\
\hline Pleomele & 15.150 & 0.068 \\
\hline Plinia & 28.083 & 0.500 \\
\hline Pluchea & 0.100 & 2.428 \\
\hline Plumeria & 0.301 & 0.200 \\
\hline Podachaenium & 0.100 & 2.428 \\
\hline
\end{tabular}

\begin{tabular}{|c|c|c|}
\hline Genus & Isoprene & Monoterpene \\
\hline Podocarpus & 0.000 & 0.000 \\
\hline Poitea & 9.313 & 0.327 \\
\hline Polyalthia & 0.000 & 0.000 \\
\hline Polygala & 0.010 & 0.010 \\
\hline Polygonum & 0.100 & 0.100 \\
\hline Polyscias & 0.100 & 0.200 \\
\hline Pomaderris & 12.833 & 1.067 \\
\hline Poncirus & 0.100 & 1.450 \\
\hline Pongamia & 9.313 & 0.327 \\
\hline Pontederia & 15.150 & 0.068 \\
\hline Populus & 63.807 & 1.022 \\
\hline Posoqueria & 0.000 & 0.000 \\
\hline Potentilla & 0.000 & 0.000 \\
\hline Pouteria & 0.000 & 0.000 \\
\hline Pouzolzia & 4.971 & 0.150 \\
\hline Premna & 0.100 & 0.100 \\
\hline Prestoea & 18.180 & 0.080 \\
\hline Pritchardia & 18.180 & 0.080 \\
\hline Prockia & 0.100 & 0.100 \\
\hline Prosopis & 0.000 & 0.000 \\
\hline Prostanthera & 0.100 & 5.000 \\
\hline Protium & 8.456 & 1.100 \\
\hline Proustia & 0.100 & 2.428 \\
\hline Prumnopitys & 0.000 & 0.000 \\
\hline Prunus & 0.000 & 0.226 \\
\hline Pseudanamomis & 28.083 & 0.500 \\
\hline Pseuderanthemum & 0.118 & 0.091 \\
\hline Pseudobombax & 0.000 & 0.000 \\
\hline Pseudocydonia & 0.057 & 0.029 \\
\hline Pseudolarix & 0.100 & 2.300 \\
\hline Pseudolmedia & 8.625 & 0.133 \\
\hline Pseudopanax & 0.100 & 0.200 \\
\hline Pseudophoenix & 18.180 & 0.080 \\
\hline Pseudopiptadeni & 9.313 & 0.327 \\
\hline Pseudosasa & 0.002 & 0.006 \\
\hline Pseudotsuga & 0.000 & 0.730 \\
\hline Psidium & 28.083 & 0.500 \\
\hline Psoralea & 9.313 & 0.327 \\
\hline Psoralidium & 9.313 & 0.327 \\
\hline Psorothamnus & 9.313 & 0.327 \\
\hline
\end{tabular}


Table 30.-Continued

\begin{tabular}{|c|c|c|}
\hline Genus & Isoprene & Monoterpene \\
\hline Psychotria & 0.000 & 0.000 \\
\hline Ptelea & 0.100 & 1.450 \\
\hline Pteralyxia & 0.100 & 0.200 \\
\hline Pterocarpus & 9.313 & 0.327 \\
\hline Pterocarya & 0.100 & 2.300 \\
\hline Pterostyrax & 0.050 & 0.050 \\
\hline Ptychosperma & 18.180 & 0.080 \\
\hline Pueraria & 8.450 & 0.600 \\
\hline Punica & 0.000 & 0.000 \\
\hline Purshia & 0.000 & 0.029 \\
\hline Pyracantha & 0.000 & 0.000 \\
\hline Pyrularia & 6.741 & 0.415 \\
\hline Pyrus & 0.000 & 0.600 \\
\hline Qualea & 5.550 & 0.050 \\
\hline Quararibea & 0.000 & 0.000 \\
\hline Quassia & 0.000 & 0.000 \\
\hline Quercus & 45.532 & 8.940 \\
\hline Quillaja & 0.057 & 0.029 \\
\hline Radermachera & 0.275 & 0.075 \\
\hline Randia & 0.000 & 0.000 \\
\hline Rapanea & 6.648 & 0.114 \\
\hline Raphiolepis & 0.000 & 0.000 \\
\hline Rauvolfia & 0.000 & 0.200 \\
\hline Ravenala & 0.000 & 0.068 \\
\hline Ravenea & 18.180 & 0.080 \\
\hline Ravenia & 0.100 & 1.450 \\
\hline Remya & 0.100 & 2.428 \\
\hline Reynoldsia & 0.100 & 0.200 \\
\hline Rhamnus & 37.000 & 0.000 \\
\hline Rhaphiolepis & 0.057 & 0.029 \\
\hline Rhapidophyllum & 18.180 & 0.080 \\
\hline Rhapis & 18.180 & 0.080 \\
\hline Rhizophora & 0.100 & 0.100 \\
\hline Rhodamnia & 28.083 & 0.500 \\
\hline Rhododendron & 0.000 & 0.100 \\
\hline Rhodomyrtus & 28.083 & 0.500 \\
\hline Rhodotypos & 0.057 & 0.029 \\
\hline Rhopalostylis & 18.180 & 0.080 \\
\hline Rhus & 0.000 & 0.000 \\
\hline Rhytidophyllum & 0.118 & 0.091 \\
\hline
\end{tabular}

\begin{tabular}{|c|c|c|}
\hline Genus & Isoprene & Monoterpene \\
\hline Ribes & 0.000 & 0.000 \\
\hline Ricinus & 0.000 & 0.133 \\
\hline Rinorea & 0.010 & 0.010 \\
\hline Robinia & 35.000 & 4.700 \\
\hline Rochefortia & 0.000 & 0.000 \\
\hline Rolandra & 0.100 & 2.428 \\
\hline Rollinia & 0.000 & 0.000 \\
\hline Rondeletia & 0.000 & 0.000 \\
\hline Rosa & 0.000 & 0.000 \\
\hline Rosmarinus & 0.010 & 2.200 \\
\hline Rothmannia & 0.000 & 0.000 \\
\hline Roupala & 0.100 & 0.200 \\
\hline Rourea & 0.048 & 0.028 \\
\hline Roystonea & 18.180 & 0.080 \\
\hline Rubus & 0.013 & 0.127 \\
\hline Rudgea & 0.000 & 0.000 \\
\hline Ruprechtia & 0.100 & 0.100 \\
\hline Rustia & 0.000 & 0.000 \\
\hline Sabal & 14.000 & 0.100 \\
\hline Sabicea & 0.000 & 0.000 \\
\hline Sadleria & 6.424 & 0.068 \\
\hline Sageretia & 12.833 & 1.067 \\
\hline Sagraea & 6.741 & 0.050 \\
\hline Salazaria & 0.100 & 5.000 \\
\hline Salix & 58.005 & 0.315 \\
\hline Salmea & 0.100 & 2.428 \\
\hline Salvia & 0.100 & 5.000 \\
\hline Samanea & 9.313 & 0.327 \\
\hline Sambucus & 0.000 & 0.000 \\
\hline Samyda & 0.100 & 0.100 \\
\hline Sanchezia & 0.118 & 0.091 \\
\hline Santalum & 6.741 & 0.415 \\
\hline Sapindus & 0.000 & 0.000 \\
\hline Sapium & 0.100 & 0.200 \\
\hline Sarcobatus & 0.100 & 0.100 \\
\hline Sarcomelicope & 0.100 & 1.450 \\
\hline Sassafras & 0.100 & 0.100 \\
\hline Savia & 0.067 & 0.133 \\
\hline Saxegothaea & 0.000 & 0.000 \\
\hline Scaevola & 0.078 & 0.620 \\
\hline
\end{tabular}


Table 30.-Continued

\begin{tabular}{|c|c|c|}
\hline Genus & Isoprene & Monoterpene \\
\hline Schaefferia & 0.100 & 0.200 \\
\hline Schefflera & 0.100 & 0.200 \\
\hline Schiedea & 0.100 & 0.100 \\
\hline Schinus & 0.000 & 7.050 \\
\hline Schisandra & 0.083 & 0.167 \\
\hline Schizolobium & 9.313 & 0.327 \\
\hline Schizomeria & 0.048 & 0.028 \\
\hline Schlegelia & 0.275 & 0.075 \\
\hline Schleichera & 44.900 & 0.000 \\
\hline Schoepfia & 6.741 & 0.415 \\
\hline Schotia & 9.313 & 0.327 \\
\hline Schradera & 0.000 & 0.000 \\
\hline Sciadopitys & 2.071 & 1.871 \\
\hline Scolosanthus & 0.000 & 0.000 \\
\hline Sebastiania & 0.067 & 0.133 \\
\hline Securidaca & 0.010 & 0.010 \\
\hline Sedum & 0.048 & 0.028 \\
\hline Seguieria & 0.100 & 0.100 \\
\hline Senefeldera & 0.067 & 0.133 \\
\hline Senegalia & 9.927 & 0.327 \\
\hline Senna & 0.000 & 0.327 \\
\hline Sequoia & 0.000 & 3.000 \\
\hline Sequoiadendron & 0.000 & 3.000 \\
\hline Serenoa & 35.000 & 0.100 \\
\hline Serianthes & 9.313 & 0.327 \\
\hline Serissa & 0.000 & 0.000 \\
\hline Sesbania & 9.313 & 0.327 \\
\hline Severinia & 0.100 & 1.450 \\
\hline Shepherdia & 0.100 & 0.200 \\
\hline Shorea & 0.000 & 0.000 \\
\hline Sida & 0.000 & 0.000 \\
\hline Sideroxylon & 0.000 & 0.000 \\
\hline Simaba & 0.000 & 0.000 \\
\hline Simarouba & 0.000 & 0.000 \\
\hline Simira & 0.000 & 0.000 \\
\hline Simmondsia & 6.741 & 0.133 \\
\hline Siparuna & 0.083 & 0.167 \\
\hline Siphoneugena & 28.083 & 0.500 \\
\hline Sloanea & 0.000 & 0.000 \\
\hline Smilax & 15.150 & 0.068 \\
\hline
\end{tabular}

\begin{tabular}{|c|c|c|}
\hline Genus & Isoprene & Monoterpene \\
\hline Solanum & 0.000 & 0.120 \\
\hline Sophora & 34.000 & 0.200 \\
\hline Sorbaria & 0.000 & 0.000 \\
\hline Sorbus & 0.333 & 1.500 \\
\hline Sorghum & 0.002 & 0.006 \\
\hline Sorocea & 8.625 & 0.133 \\
\hline Sparattosperma & 0.275 & 0.075 \\
\hline Spartium & 8.080 & 0.473 \\
\hline Spathodea & 0.275 & 0.075 \\
\hline Spiraea & 0.000 & 0.000 \\
\hline Spondias & 0.000 & 2.260 \\
\hline Stachytarpheta & 0.100 & 0.100 \\
\hline Stahlia & 9.313 & 0.327 \\
\hline Staphylea & 0.100 & 1.600 \\
\hline Stegnosperma & 0.100 & 0.100 \\
\hline Stenocarpus & 0.100 & 0.200 \\
\hline Stenocereus & 0.100 & 0.100 \\
\hline Stenogyne & 0.100 & 5.000 \\
\hline Stephanandra & 0.057 & 0.029 \\
\hline Sterculia & 0.000 & 0.000 \\
\hline Stewartia & 0.000 & 0.000 \\
\hline Stifftia & 0.100 & 2.428 \\
\hline Stillingia & 0.067 & 0.133 \\
\hline Streblus & 8.625 & 0.133 \\
\hline Strelitzia & 15.150 & 0.068 \\
\hline Streptosolen & 0.078 & 0.120 \\
\hline Strobilanthes & 0.118 & 0.091 \\
\hline Strumpfia & 0.000 & 0.000 \\
\hline Strychnos & 0.000 & 0.200 \\
\hline Stylogyne & 6.648 & 0.114 \\
\hline Styphelia & 6.648 & 0.157 \\
\hline Styrax & 0.000 & 0.100 \\
\hline Suriana & 0.048 & 0.028 \\
\hline Swartzia & 9.313 & 0.327 \\
\hline Sweetia & 9.313 & 0.327 \\
\hline Swietenia & 0.100 & 0.100 \\
\hline Syagrus & 18.180 & 0.080 \\
\hline Symphoricarpos & 1.000 & 0.200 \\
\hline Symphysia & 0.086 & 0.157 \\
\hline Symplocos & 0.050 & 0.050 \\
\hline
\end{tabular}


Table 30.-Continued

\begin{tabular}{|c|c|c|}
\hline Genus & Isoprene & Monoterpene \\
\hline Syncarpia & 28.083 & 0.500 \\
\hline Syringa & 0.000 & 0.100 \\
\hline Syzygium & 12.100 & 0.000 \\
\hline Tabebuia & 0.000 & 0.000 \\
\hline Tabernaemontana & 0.100 & 0.200 \\
\hline Tachigali & 9.313 & 0.327 \\
\hline Taiwania & 0.086 & 0.714 \\
\hline Tamarindus & 9.313 & 0.327 \\
\hline Tamarix & 0.000 & 0.040 \\
\hline Tamonea & 0.100 & 0.100 \\
\hline Tapirira & 0.000 & 2.260 \\
\hline Tasmannia & 2.660 & 1.200 \\
\hline Taxodium & 0.000 & 3.000 \\
\hline Taxus & 0.100 & 1.500 \\
\hline Tecoma & 0.000 & 0.000 \\
\hline Tectona & 0.100 & 0.100 \\
\hline Telopea & 0.100 & 0.200 \\
\hline Templetonia & 9.313 & 0.327 \\
\hline Tephrosia & 9.313 & 0.327 \\
\hline Terminalia & 5.550 & 0.050 \\
\hline Ternstroemia & 0.000 & 0.000 \\
\hline Tetracoccus & 0.067 & 0.133 \\
\hline Tetradium & 0.100 & 1.450 \\
\hline Tetragastris & 8.456 & 1.100 \\
\hline Tetramolopium & 0.100 & 2.428 \\
\hline Tetrapanax & 0.100 & 0.200 \\
\hline Tetraplasandra & 0.100 & 0.200 \\
\hline Tetrapterys & 0.010 & 0.010 \\
\hline Tetrazygia & 5.550 & 0.050 \\
\hline Tetrorchidium & 0.067 & 0.133 \\
\hline Teucrium & 0.100 & 5.000 \\
\hline Theobroma & 0.000 & 0.000 \\
\hline Thespesia & 0.000 & 0.000 \\
\hline Thevetia & 0.100 & 0.200 \\
\hline Thouinia & 44.900 & 0.000 \\
\hline Thuja & 0.008 & 0.071 \\
\hline Thujopsis & 0.086 & 0.714 \\
\hline Thunbergia & 0.118 & 0.091 \\
\hline Tibouchina & 5.550 & 0.050 \\
\hline Tilia & 0.000 & 0.000 \\
\hline
\end{tabular}

\begin{tabular}{|c|c|c|}
\hline Genus & Isoprene & Monoterpene \\
\hline Timonius & 0.000 & 0.000 \\
\hline Tipuana & 9.313 & 0.327 \\
\hline Toona & 0.000 & 0.100 \\
\hline Torralbasia & 0.100 & 0.200 \\
\hline Torreya & 0.000 & 1.500 \\
\hline Touchardia & 4.971 & 0.150 \\
\hline Tournefortia & 0.000 & 0.000 \\
\hline Tovomita & 0.000 & 0.000 \\
\hline Toxicocendron & 0.000 & 2.260 \\
\hline Toxicodendron & 0.000 & 0.000 \\
\hline Trachycarpus & 14.000 & 0.100 \\
\hline Trema & 0.000 & 0.167 \\
\hline Trematolobelia & 0.078 & 0.620 \\
\hline Triadica & 0.067 & 0.133 \\
\hline Trichilia & 0.100 & 0.100 \\
\hline Triphasia & 0.100 & 1.450 \\
\hline Tristaniopsis & 28.083 & 0.500 \\
\hline Trithrinax & 0.000 & 0.080 \\
\hline Triumfetta & 0.000 & 0.000 \\
\hline Trixis & 0.100 & 2.428 \\
\hline Trophis & 8.625 & 0.133 \\
\hline Tsuga & 0.015 & 0.360 \\
\hline Tupidanthus & 0.100 & 0.200 \\
\hline Turpinia & 0.100 & 1.600 \\
\hline Ulex & 9.516 & 1.408 \\
\hline Ulmus & 0.000 & 1.295 \\
\hline Umbellularia & 0.000 & 0.100 \\
\hline Ungnadia & 44.900 & 0.000 \\
\hline Urera & 0.000 & 0.150 \\
\hline Urvillea & 44.900 & 0.000 \\
\hline Vaccinium & 0.100 & 0.100 \\
\hline Valeriana & 0.000 & 0.000 \\
\hline Vallesia & 0.100 & 0.200 \\
\hline Vanclevea & 0.100 & 2.428 \\
\hline Vangueria & 0.000 & 0.000 \\
\hline Varronia & 0.000 & 0.000 \\
\hline Vauquelinia & 0.057 & 0.029 \\
\hline Veitchia & 18.180 & 0.080 \\
\hline Ventilago & 12.833 & 1.067 \\
\hline Vernicia & 0.067 & 0.133 \\
\hline
\end{tabular}


Table 30.-Continued

\begin{tabular}{|l|r|c|}
\hline Genus & Isoprene & Monoterpene \\
\hline Verticordia & 28.083 & 0.500 \\
\hline Viburnum & 0.000 & 0.000 \\
\hline Viminaria & 28.083 & 0.500 \\
\hline Virgilia & 9.313 & 0.327 \\
\hline Virola & 13.000 & 1.200 \\
\hline Vitex & 0.000 & 0.100 \\
\hline Vitis & 0.000 & 0.310 \\
\hline Vochysia & 5.550 & 0.050 \\
\hline Wallenia & 6.648 & 0.114 \\
\hline Waltheria & 0.000 & 0.000 \\
\hline Washingtonia & 12.500 & 0.000 \\
\hline Wedelia & 0.100 & 2.428 \\
\hline Weigela & 0.000 & 0.000 \\
\hline Weinmannia & 0.048 & 0.028 \\
\hline Wigandia & 0.078 & 0.120 \\
\hline Wikstroemia & 5.550 & 0.050 \\
\hline Wilkesia & 0.100 & 2.428 \\
\hline Wisteria & 0.100 & 0.200 \\
\hline Wodyetia & 18.180 & 0.080 \\
\hline
\end{tabular}

\begin{tabular}{|l|r|c|}
\hline Genus & Isoprene & Monoterpene \\
\hline Wollemia & 0.100 & 1.500 \\
\hline Wrightia & 0.100 & 0.200 \\
\hline Xanthostemon & 28.083 & 0.500 \\
\hline Ximenia & 6.741 & 0.415 \\
\hline Xylococcus & 0.086 & 0.157 \\
\hline Xylomelum & 0.100 & 0.200 \\
\hline Xylopia & 0.000 & 0.000 \\
\hline Xylosma & 8.000 & 0.000 \\
\hline Yucca & 0.000 & 0.068 \\
\hline Zanthoxylum & 0.100 & 1.450 \\
\hline Zapoteca & 9.313 & 0.327 \\
\hline Zea & 0.002 & 0.006 \\
\hline Zelkova & 0.000 & 0.200 \\
\hline Zenobia & 0.086 & 0.157 \\
\hline Zeyheria & 0.275 & 0.075 \\
\hline Zieria & 0.100 & 1.450 \\
\hline Ziziphus & 12.833 & 1.067 \\
\hline Zollernia & 9.313 & 0.327 \\
\hline
\end{tabular}

